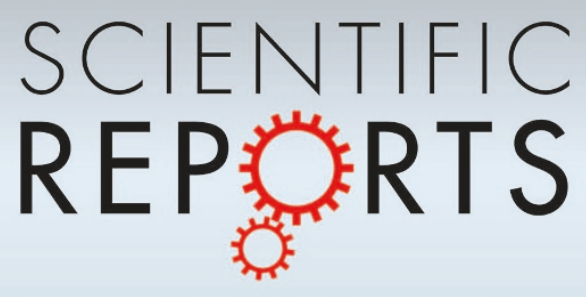

OPEN

SUBJECT AREAS:

TRANSCRIPTIONAL REGULATORY ELEMENTS

CELL FATE AND CELL LINEAGE

Received

30 October 2013

Accepted

24 April 2014

Published

12 May 2014

Correspondence and requests for materials should be addressed to

H.M. (miyazawah@

kph.bunri-u.ac.jp)

\section{Tal2 expression is induced by all-trans retinoic acid in P19 cells prior to acquisition of neural fate}

\author{
Takanobu Kobayashi', Rie Komori' ', Kiyoshi Ishida' ', Katsuhito Kino', Sei-ichi Tanuma² \\ \& Hiroshi Miyazawa'
}

\begin{abstract}
${ }^{1}$ Kagawa School of Pharmaceutical Sciences, Tokushima Bunri University, 1314-1 Shido, Sanuki, Kagawa 769-2193, Japan, ${ }^{2}$ Department of Biochemistry, Faculty of Pharmaceutical Sciences, Tokyo University of Science, 2641 Yamazaki, Noda, Chiba 278-8510, Japan.
\end{abstract}

TAL2 is a member of the basic helix-loop-helix family and is essential for the normal development of the mouse brain. However, the function of TAL2 during brain development is unclear. P19 cells are pluripotent mouse embryonal carcinoma cells that adopt neural fates upon exposure to all-trans retinoic acid (atRA) and culture in suspension. We found that the expression of Tal2 gene was induced in P19 cells after addition of atRA in suspension culture. Tal2 expression was detected within $3 \mathrm{~h}$ after the induction, and had nearly returned to basal levels by $24 \mathrm{~h}$. When GFP-tagged TAL2 (GFP-TAL2) was expressed in P19 cells, we observed GFP-TAL2 in the nucleus. Moreover, we showed that atRA and retinoic acid receptor $\alpha$ regulated $\mathrm{Tal} 2$ expression. These results demonstrate for the first time that atRA induces Tal2 expression in P19 cells, and suggest that TAL2 commits to the acquisition of neural fate in brain development. 


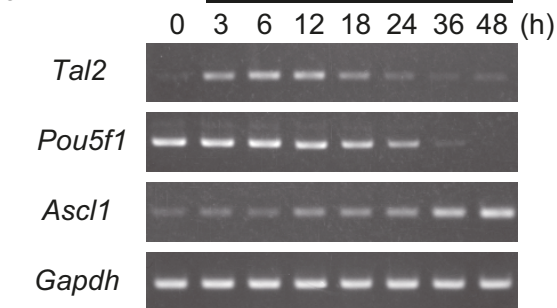

b

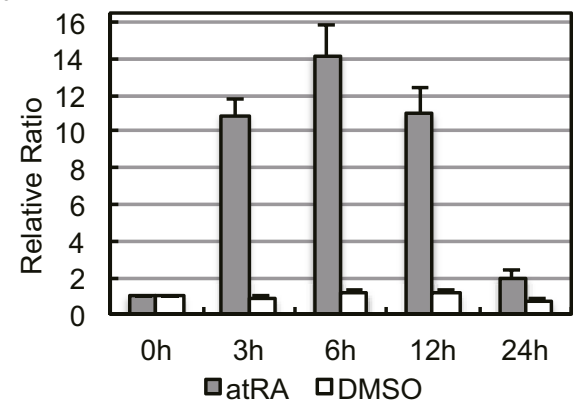

C

Tal2

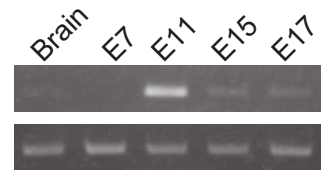

Figure $1 \mid$ Tal2 was transiently expressed in P19 cells introduced into the neural differentiation and in embryogenesis. P19 cells were treated with $1 \mu \mathrm{M}$ atRA in suspension culture. (a) RT-PCR was performed to examine Tal2, Pouff1 (the pluripotent transcription factor), Ascl1 (the neurogenic bHLH transcription factor), and Gapdh expression in P19 cells at 0, 3, 6, 12, 18, 24, 36 and $48 \mathrm{~h}$. These images were cropped. Full-length gels are presented in Supplementary Figure S2. (b) Real-time PCR was performed to measure Tal2 expression at $0,3,6,12$ and $24 \mathrm{~h}$. The gene expression levels of Tal2 were normalized using Hmbs gene as an internal standard. Fold expression was defined as fold increase relative to $0 \mathrm{~h}$. Data represent the mean \pm S.E. of three independent experiments. (c) RT-PCR was performed to examine Tal2 expression in mouse embryogenesis (E7, E11, E15 and E17) and adult brain. Tal2 expression was evident at E11. These images were cropped. Full-length gels are presented in Supplementary Figure S3.

development, we have attempted to identify genes that commit to the neural fate using P19 cells that were introduced into the neural differentiation. In these studies, we found that Tal2 expression was immediately upregulated in P19 cells. Here, we investigated the relationship of atRA, cell aggregation, and Tal2 induction in P19 cells.

\section{Results}

Tal2 was transiently expressed in P19 cells introduced into the neural differentiation. We found that Tal2 expression was

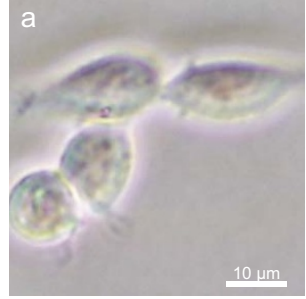

Phase-contrast

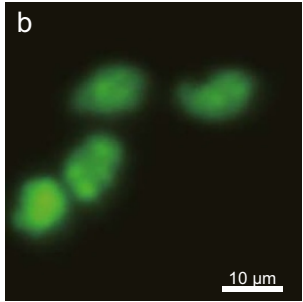

GFP-TAL2

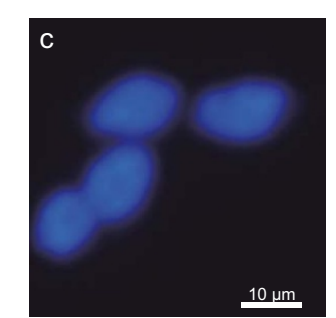

Hoechst33258

upregulated in P19 cells introduced into the neural differentiation for our study using DNA microarray experiments (unpublished data). To study the alteration of Tal2 expression in more detail here, we extracted RNA from P19 cells 0, 3, 6, 12, 18, 24, 36 and $48 \mathrm{~h}$ after addition of atRA in suspension culture, and investigated Tal2 expression in P19 cells (Fig. 1a). We also examined the expression of Pou $5 f 1$ gene (also known as Oct3/4), which encodes the transcription factor in pluripotent cells, and Ascl1 gene (also known as Mash1), which encodes a neurogenic bHLH transcription factor, to assess whether P19 cells were induced to neural cell fate. Pou5f1 expression decreases during neural differentiation of P19 cells ${ }^{26,27}$; contemporaneously, Ascl1 expression increases ${ }^{27,28}$. As previously reported, Pou5f1 expression decreased and Ascll expression increased contemporaneously in atRA-treated, aggregated P19 cells. This observation demonstrated that P19 cells lost the pluripotency by addition of atRA in suspension culture and were entered into neural cell fate. Tal2 expression increased within $3 \mathrm{~h}$ after treatment, and Tal2 mRNA levels was returned to the basal levels within $24 \mathrm{~h}$ after treatment. In contrast, the expression of all three genes-Tal2, Pou5f1, and Ascl1was not altered by DMSO treatment in suspension culture (see Supplementary Fig. S1 online).

Next, we used real-time PCR assays to measure Tal2 expression in P19 cells (Fig. 1b). Tal2 expression increased $3 \mathrm{~h}$ and $6 \mathrm{~h}$ after treatment. A reduction in Tal2 expression from peak levels was first observed at $12 \mathrm{~h}$ and Tal2 expression was close to the basal levels at $24 \mathrm{~h}$. These results indicate that Tal2 expression is altered in P19 cells for one day.

It has been reported that Tal2 expression was observed in embryonic head (E10.5 to E14.5) ${ }^{10}$. We also confirmed Tal2 expression in embryogenesis (Fig. 1c). For this purpose, we used a mouse cDNA panel and RT-PCR. As previously reported, Tal2 expression was evident at E11 and was not observed in adult brain. In addition, Tal2 was also hardly observed in E7, E15 and E17. The transient expression of Tal2 was confirmed in mouse embryogenesis, as well as Tal2 transiently expressed in P19 cells by addition of atRA in suspension culture.

Localization of TAL2 in P19 cells. To examine the behavior of TAL2 in P19 cells, we generated GFP-tagged TAL2 (GFP-TAL2) expression construct. This construct was introduced into P19 cells via transfection, and these cells were stained with Hoechst33258 $48 \mathrm{~h}$ post transfection. We then used fluorescence microscopy to visualize these cells (Fig. 2). GFP-TAL2 expressed in P19 cells was detected in nuclei.

atRA induced the expression of Tal2 gene in P19 cells. atRA and cell aggregation are important for neural differentiation of P19 cells ${ }^{12-14}$. Thus, we examined how these treatments were involved in the expression of Tal2 gene. When P19 cells were treated with atRA and cultured as a monolayer in culture dishes, Tal2 expression was nearly the same as that in atRA-treated and cell-aggregated P19 cells (Fig. 3a). Additionally, the Tal2 gene was not induced by DMSO

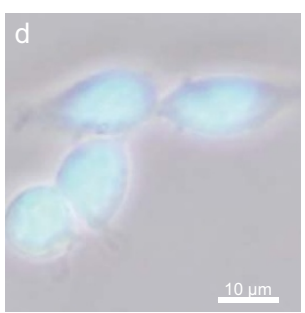

Overlay

Figure $2 \mid$ The localization of GFP-TAL2 visualized by fluorescence microscopy. P19 cells were transfected with the expression vector encoding GFPTAL2 and were stained with Hoechst33258 $48 \mathrm{~h}$ after transfection. (a) Phase-contrast. (b) GFP-TAL2. (c) Hoechst33258. (d) Overlay. 
treatment in suspension culture of P19 cells (see Supplementary Fig. S1 online). These results indicate that the induction of Tal2 in P19 cells is associated with atRA.

atRA binds to the RARs (RAR $\alpha, \beta$, or $\gamma$ ), and RARs form heterodimers with RXRs (RXR $\alpha, \beta$, or $\gamma)$. atRA-bound RAR/RXR heterodimer can regulate the transcription of target genes. To elucidate the involvement of these receptors in Tal2 induction, we confirmed the expression of Rar genes and Rxr genes in P19 cells after addition of atRA in suspension culture (Fig. 3b). For these six genes, the expression of Rara, Rxra or Rxrb was not altered at $48 \mathrm{~h}$. The expression of Rarg and Rxrg had decreased relative to basal levels. On the other hand, Rarb expression was not evident at $0 \mathrm{~h}$, but it was evident within $3 \mathrm{~h}$ after treatment, as it has been reported that Rarb was induced by retinoic acid in P19 cells ${ }^{29,30}$. The induction of Tal2 and Rarb were similar in P19 cells, but Rarb expression was not altered from $3 \mathrm{~h}$ to $48 \mathrm{~h}$ while the expression of Tal2 gene had decreased from peak levels by $24 \mathrm{~h}$.

a

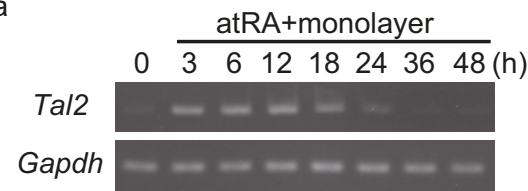

b

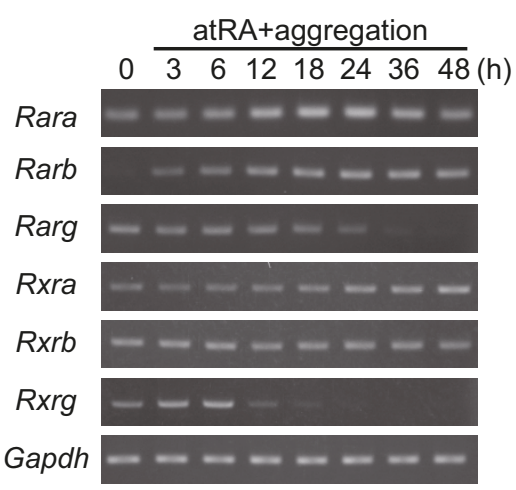

Next, in order to investigate the relation between atRA and its receptors, we used four agonists instead of atRA; Am80 ${ }^{31}$ (RAR $\alpha$ agonist), adapalene ${ }^{32}$ (RAR $\beta$ and RAR $\gamma$ agonist), AC-41848 (RAR $\gamma$ agonist) and methoprene acid $^{33}$ (RXR pan-agonist). Am 80 was able to induce Tal2 gene as well as atRA. Adapalene also induced Tal2 in P19 cells, but the induction of Tal2 by adapalene was weaker than that by atRA or Am80. In contrast, neither AC-41848 nor methoprene acid induced Tal2 expression (Fig. 3c). Moreover, We also assessed morphological changes of P19 cells that were treated with atRA, DMSO, or each agonist (Fig. 3d). P19 cells formed spherical aggregates called embryoid bodies in the presence of atRA, Am80 or adapalene. Whereas, DMSO, AC-41848 or methoprene acid did not formed embryoid bodies.

Tal 2 expression was induced by RAR $\alpha$. Adapalene formed P19 cells into embryoid bodies, and weakly induced the expression of Tal2 compared with atRA or Am80. Moreover, it has been reported that

C

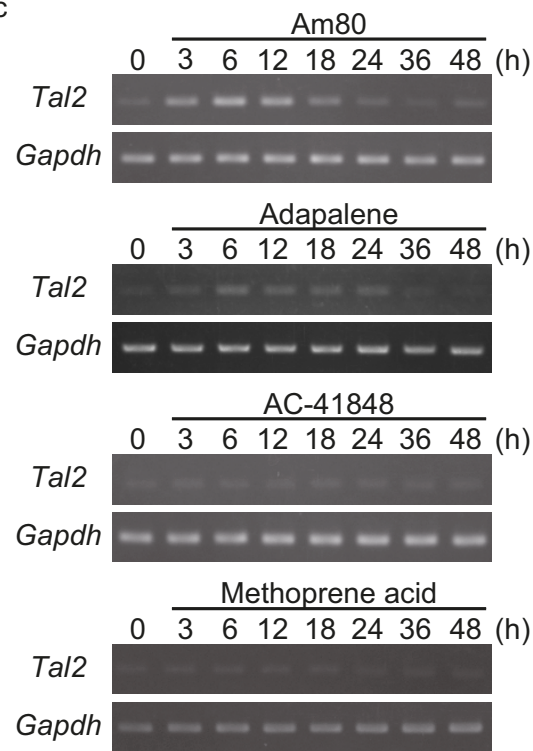

d

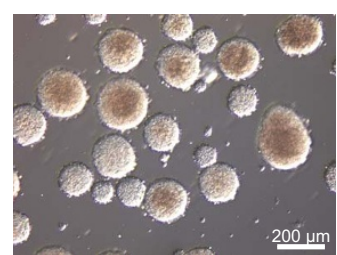

atRA

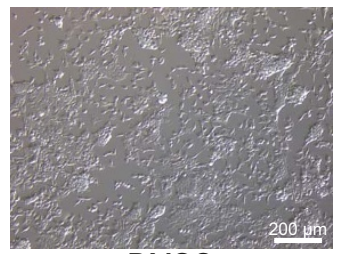

DMSO

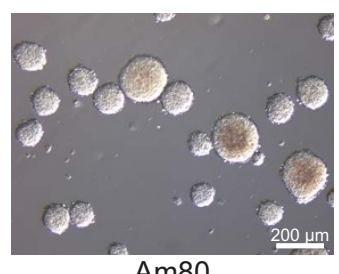

Am80

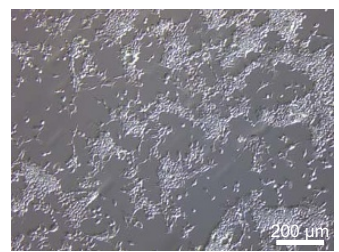

AC-41848

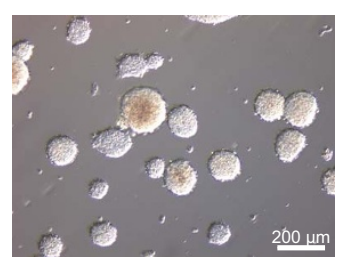

Adapalene

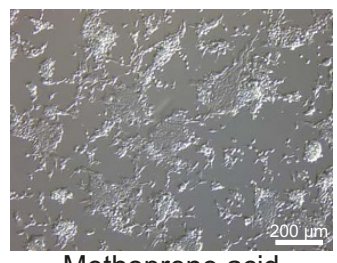

Methoprene acid

Figure 3 atRA were involeved in Tal2 expression in P19 cells. (a) P19 cells were treated with $1 \mu \mathrm{M}$ atRA and cultured in adherent conditions for 0 , 3, 6 , 12, 18, 24, 36 and $48 \mathrm{~h}$. RT-PCR was performed to examine Tal2 expression in these cells. Tal2 expression was induced by atRA treatment of cells in adherent conditions as well as those in suspension culture. These images were cropped. Full-length gels are presented in Supplementary Figure S4. (b) The expression of Rar genes and Rxr genes in P19 cells after addition of atRA in suspension culture. These images were cropped. Full-length gels are presented in Supplementary Figure S5. (c) RT-PCR was performed to examine Tal2 expression in P19 cells that were treated with agonists in suspension culture-Am80 (RAR $\alpha$ agonist), adapalene (RAR $\beta$ and RAR $\gamma$ agonist), AC-41848 (RAR $\gamma$ agonist) or methoprene acid (RXR pan-agonist). These images were cropped. Full-length gels are presented in Supplementary Figure S6. (d) Morphology of P19 cells following treatment with atRA, DMSO, or each or four agonists for $48 \mathrm{~h}$. 
adapalene has stimulated RAR $\alpha$ weakly ${ }^{32}$. Although the expression of Rarb gene was not observed at $0 \mathrm{~h}$ in $\mathrm{P} 19$ cells, RAR $\alpha$ protein was expressed at $0 \mathrm{~h}$. Based on these findings, we assumed that RAR $\alpha$ had an intimate involvement in Tal2 induction in P19 cells.

We used RNA interference to suppress RAR $\alpha$ expression in P19 cells, and examine the involvement of RAR $\alpha$ and the induction of Tal2. The RNAi-mediated suppression of RAR $\alpha$ in P19 cells was verified by Western blotting (Fig. 4a). Tal2 expression significantly decreased by RAR $\alpha$ suppression in P19 cells after addition of atRA in suspension culture (Fig. $4 \mathrm{~b}$ ). These results indicate that atRA-RAR $\alpha$ complexes induce Tal2 gene in P19 cells.

\section{Discussion}

P19 EC cells are induced into neural differentiation by addition of atRA in suspension culture ${ }^{13,14}$. We explore the molecular mechanisms of the entry into neural differentiation using P19 cells. We found that Tal2 expression was altered rapidly and transiently in these cells (Fig. 1a and b). Interestingly, the transient expression of Tal2 gene was observed at the stage of organogenesis during embryonic development (Fig. 1c). Tal2 expression in embryonic head was reported previously ${ }^{10,11}$, and TAL2 is inferred to play a role at the early stage of brain development. Because P19 cells have been used as model cells for the studies of neural differentiation, a detailed investigation of the molecular mechanisms regulating Tal2 expression in P19 cells may be key to understanding the function of TAL2 in brain development.

We investigated the correlation of atRA, cell aggregation and Tal2 induction in P19 cells. As a result, atRA participated in Tal2 induction in P19 cells, but cell aggregation was not necessary for Tal2 induction (Fig. 3a). Next, we examined the involvement of RAR/ RXR in the induction of Tal2 in P19 cells (Fig. 3c). Am80, a RAR $\alpha$ agonist, was able to induce Tal2 expression in P19 cells. Adapalene also weakly induced Tal2 in P19 cells. Although adapalene is an

a

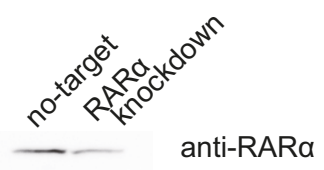

anti- $\beta$-actin

b

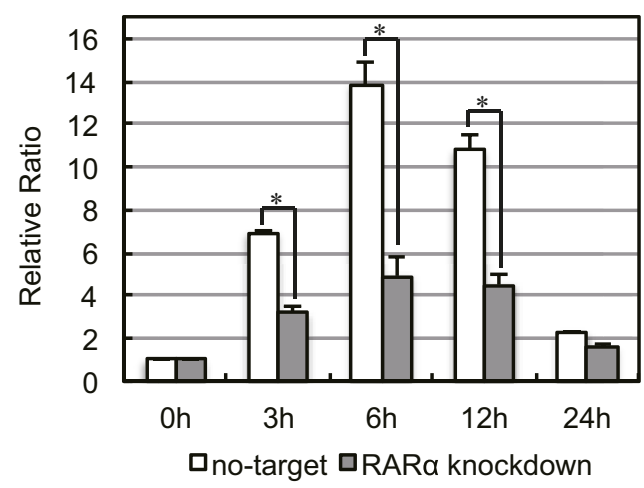

Figure 4 | Tal2 expression was suppressed by RAR $\alpha$ knockdown. (a) The suppression of RAR $\alpha$ in P19 cells was verified by Western blotting. The protein expression levels were normalized using $\beta$-actin as an internal standard, and RAR $\alpha$ expression was examined under the same conditions as $\beta$-actin. These images were cropped. Full-length gels are presented in Supplementary Figure S7. (b) Real-time PCR was performed to measure Tal2 expression in P19 cells subjected to RNAi-mediated RAR $\alpha$ knockdown. A significant reduction of Tal2 expression was observed following RAR $\alpha$ knockdown. Data represent the mean \pm S.E. of three independent experiments. ${ }^{*}, p<0.05$. agonist of $\operatorname{RAR} \beta$ and $\operatorname{RAR} \gamma$, it might also react to RAR $\alpha$ in P19 cells. Indeed, $R A R \alpha$ is less responsive to adapalene than $\operatorname{RAR} \beta$ and $\operatorname{RAR} \gamma$, and $R X R \alpha$ is no responsive to adapalene ${ }^{32}$. Rarb gene was not expressed in P19 cells at $0 \mathrm{~h}$, and was induced by atRA as well as Tal2 gene. Therefore, adapalene is thought to stimulate the RAR $\alpha$ signaling weakly and to induce the expression of Tal2 in P19 cells. Moreover, RAR $\alpha$ knock down by RNA interference suppressed Tal2 expression in P19 cells (Fig. 4b). These results indicate that atRARAR $\alpha$ complexes induce transcription of Tal2 gene in P19 cells.

The embryoid bodies were formed by the agonists, atRA, Am80 and adapalene, that induced the expression of Tal2 in P19 cells (Fig. 3d). These results raise the possibility that TAL2 is also involved in the formation of embryoid bodies of P19 cells. TAL2 induced by these agonists might activate genes such as $F g f 8^{34}$ as a transcription factor that are involved in cell aggregation.

The bHLH family to which TAL2 belongs regulates a variety of biological processes. The bHLH usually has two functionally distinct domains-the basic domain and the HLH domain. The bHLH proteins form dimers via HLH domains and bind DNA via the basic domains. These dimers activate target genes in the nucleus ${ }^{2}$. Therefore, TAL2, which is a bHLH protein, is probably involved in transcriptional activation of target genes in the nucleus. Indeed, the GFP-TAL2 fusion protein that we generated localized to nuclei (Fig. 2). Moreover, GFP-TAL2 was highly enriched in a part of each nucleus. In zebrafish, TAL2 is expressed in the lateral floor plate of the spinal cord ${ }^{35}$. The floor plate is an important signal center that specifies neurons and glia cells in the ventral neural tube and guides the trajectory of outgrowing axons. TAL2 acts upstream of the gad67 gene that is the GABA-synthesizing enzyme glutamic acid decarboxylase in Kolmer-Agduhr" $\left(\mathrm{KA}^{\prime \prime}\right)$ cells of the lateral floor plate ${ }^{36}$. However, it is not clear whether the mammalian spinal cord has KA" cells, and Tal2 has not yet been observed in the mouse spinal cord.

atRA is important for embryogenesis and the differentiation of the nervous system, and we demonstrated for the first time that Tal2 expression was induced by atRA, which introduced P19 cells into neural differentiation. In addition, the transient expression of Tal2 was observed in organogenesis of development. These findings suggest that TAL2 plays a role in acquisition of neural cell fate as a transcription factor in neural differentiation. Because the neural differentiation of P19 cells in part mimics the development of the nervous system, P19 cells are useful for studying the molecular mechanism of TAL2 in neural differentiation. Further work is underway to clarify the function of TAL2 in neural differentiation using the differentiation system of P19 cells.

\section{Methods}

Cell culture and neural differentiation. P19C6, a subclone of P19 mouse embryonic carcinoma cell line, was used in this study ${ }^{37}$. P19C6 was provided by the RIKEN BRC through the National Bio-Resource Project of the MEXT, Japan. P19 cells were cultured in $\alpha$-MEM (Sigma-Aldrich, MO) supplemented with 10\% FBS (Life Technologies, CA) and $2 \mathrm{mM}$ L-glutamine (Kanto Chemical, Japan). To induce neural differentitation, these cells were aggregated in a suspension culture dish (SUMILON, Japan) at a seeding density of $2 \times 10^{5} \mathrm{cell} / \mathrm{ml}$ in the presence of $1 \mu \mathrm{M}$ atRA (Sigma-Aldrich), which was dissolved in DMSO (Sigma-Aldrich) ${ }^{28}$. DMSO concentration in culture condition was $0.01 \%$. To identify the RAR and RXR subtypes, $100 \mathrm{nM}$ Am80 (RAR $\alpha$ agonist), $100 \mathrm{nM}$ adapalene (RAR $\beta$ and RAR $\gamma$ agonist), $100 \mathrm{nM}$ AC-41848 (RAR $\gamma$ agonist), or $100 \mathrm{nM}$ methoprene acid (RXR panagonist) were used in place of atRA ${ }^{28,29}$. These agonists were purchased from SigmaAldrich.

RT-PCR and real-time PCR. RNeasy Mini Kit (QIAGEN, Germany) was used accoding to the manufacturer's instructions to isolate total RNA from P19 cells. RTPCR was performed as described previously ${ }^{27}$. In brief, reverse transcription was performed with $1 \mu \mathrm{g}$ of total RNA and SuperScript III reverse transcriptase (Life Technologies). AmpliTaq Gold 360 Master Mix (Life Technologies) and gene specific primer were used for amplification of target genes. The list of primers can be found as Supplementary Table S1 online. The Gapdh gene was used as an internal standard. To assess Tal2 expression in normal mouse embryogenesis, MTC Multiple Tissue cDNA Panels (TAKARA BIO, Japan) were used as templates. 
Real-time PCR was performed as described previously ${ }^{27}$. In brief, High Capacity cDNA Reverse Transcription Kit (Life Technologies) was used to synthesize cDNA from $0.5 \mu \mathrm{g}$ of total RNA. SYBR Green PCR Master Mix (Life Technologies) and 7500 Real-Time PCR system (Life Technologies) were used according to the manufacturer's protocols to perform real-time PCR. The list of primers can be found as Supplementary Table S2 online. The hydroxymethylbilane synthase ( $\mathrm{Hmbs}$ ) gene was used as an internal standard, and Tal2 expression levels were normalized to Hmbs expression levels. Although we used Hmbs as a reference gene in real-time PCR, we have confirmed the constant expression of Hmbs in P19 cells after addition of atRA in suspension culture as well as Gapdh. Fold expression was defined as fold increase relative to $0 \mathrm{~h}$.

Microscopy. GFP-fused Tal2 subcloned into pCAGGS vector ${ }^{38}$. Lipofectamine 2000 (Life Technologies) was used according to the manufacturer's instructions to transfect with the constructed vector into P19 cells. After the transfection, cells were washed with phosphate-buffered saline (PBS), and fixed via incubation with $1 \%$ glutaraldehyde (nacalai tesque, Japan) in PBS for $30 \mathrm{~min}$. After washed with PBS, the cells were stained with Hoechst 33258 for 30 min. After washed with PBS, the cells were visualized using BZ-8000 (KEYENCE, Japan). Morphological changes of P19 cells were assessed at $48 \mathrm{~h}$ after atRA, DMSO, or four agonists treatment. A relief contrast microscope (OLYMPUS, Japan) was used to examine these cells.

RNA interference. We used pSilencer 3.1-H1 (Life Technologies) as an shRNA vector. The sequences of the shRNA duplexes used RAR $\alpha$ knockdown were as follows: $R A R \alpha$ gene, sense 5' - GCAAGTACACTACGAACAACATTCAAGAGATGTTGTTCGTAGTGTACTTGCTTTTTTGGAA- ${ }^{\prime}$ and antisense 5 $^{\prime}$ - TTCCAAAAA AGCAAGTACACTACGAACAACATCTCTTGAATGTTGTTCGTAGTGTACTTGC-3', no-target as a control, sense 5'-GTACTATTCGACACGCGAAGTTCAAGAGACTTCGCGTGTCGAATAGTACTTTTTTGGAA- ${ }^{\prime}$ ' and antisense $5^{\prime}$-TTCCAAAAAAGTACTATTCGACACGCGAAGTCTCTTGAACTTCGCGTGTCGAATAGTAC-3'. Lipofectamine 2000 was used to transfect with each of these constructs into P19 cells. Western blotting probed with an antiRAR $\alpha$ antibody (Santa Cruz Biotechnology, CA) and anti-Beta-Actin antibody (Thermo SCIENTIFIC, CA) were performed to assess RAR $\alpha$ knockdown. The influence of Tal2 expression by RAR $\alpha$ suppression was examined by real-time PCR.

1. Kageyama, R., Ohtsuka, T., Hatakeyama, J. \& Ohsawa, R. Roles of bHLH genes in neural stem cell differentiation. Exp. Cell. Res. 306, 343-348 (2005).

2. Li, J. et al. Identification and analysis of the mouse basic/Helix-Loop-Helix transcription factor family. Biochem. Biophys. Res. Commun. 350, 648-656 (2006).

3. Xia, Y. et al. TAL2, a helix-loop-helix gene activated by the $(7 ; 9)(\mathrm{q} 34 ; \mathrm{q} 32)$ translocation in human T-cell leukemia. Proc. Natl. Acad. Sci. U. S. A. 88, 11416-11420 (1991).

4. Smith, S. D. et al. Clinical and biologic characterization of T-cell neoplasias with rearrangements of chromosome 7 band q34. Blood. 71, 395-402 (1988).

5. Tycko, B., Reynolds, T. C., Smith, S. D. \& Sklar, J. Consistent breakage between consensus recombinase heptamers of chromosome 9 DNA in a recurrent chromosomal translocation of human T cell leukemia. J. Exp. Med. 169, 369-377 (1989).

6. Marculescu, R. et al. Distinct $\mathrm{t}(7 ; 9)(\mathrm{q} 34 ; \mathrm{q} 32)$ breakpoints in healthy individuals and individuals with T-ALL. Nat. Genet. 33, 342-344 (2003).

7. Guo, N. L. et al. Confirmation of gene expression-based prediction of survival in non-small cell lung cancer. Clin. Cancer. Res. 14, 8213-8220 (2008).

8. $\mathrm{Zhu}, \mathrm{H}$. \& Yu, J. J. Gene expression patterns in the histopathological classification of epithelial ovarian cancer. Exp. Ther. Med. 1, 187-192 (2010).

9. Guo, Y. et al. Correlations among ERCC1, XPB, UBE2I, EGF, TAL2 and ILF3 revealed by gene signatures of histological subtypes of patients with epithelial ovarian cancer. Oncol. Rep. 27, 286-292 (2012).

10. Mori, S. et al. The leukemic oncogene tal-2 is expressed in the developing mouse brain. Brain. Res. Mol. Brain. Res. 64, 199-210 (1999).

11. Bucher, K. et al. The T cell oncogene Tal2 is necessary for normal development of the mouse brain. Dev. Biol. 227, 533-544 (2000).

12. McBurney, M. W. P19 embryonal carcinoma cells. Int. J. Dev. Biol. 37, 135-140 (1993)

13. McBurney, M. W., Jones-Villeneuve, E. M., Edwards, M. K. \& Anderson, P. J. Control of muscle and neuronal differentiation in a cultured embryonal carcinoma cell line. Nature. 299, 165-167 (1982).

14. Jones-Villeneuve, E. M., Rudnicki, M. A., Harris, J. F. \& McBurney, M. W. Retinoic acid-induced neural differentiation of embryonal carcinoma cells. Mol. Cell. Biol. 3, 2271-2279 (1983)

15. Voronova, A., Fischer, A., Ryan, T., Al Madhoun, A. \& Skerjanc, I. S. Ascl1/Mash1 is a novel target of Gli2 during Gli2-induced neurogenesis in P19 EC cells. PLoS. One. 6, e19174 (2011).

16. Alam, A. H., Suzuki, H. \& Tsukahara, T. Expression analysis of Fgf8a \&Fgf8b in early stage of P19 cells during neural differentiation. Cell. Biol. Int. 33, 1032-1037 (2009)

17. Chambon, P. A decade of molecular biology of retinoic acid receptors. FASEB. J. 10, 940-954 (1996).

18. Balmer, J. E. \& Blomhoff, R. Gene expression regulation by retinoic acid. J. Lipid. Res. 43, 1773-1808 (2002).
19. Chen, Y. \& Reese, D. H. The retinol signaling pathway in mouse pluripotent P19 cells. J. Cell. Biochem. 112, 2865-2872 (2011).

20. De Luca, L. M. Retinoids and their receptors in differentiation, embryogenesis, and neoplasia. FASEB. J. 5, 2924-2933 (1991).

21. Maden, M. Retinoic acid in the development, regeneration and maintenance of the nervous system. Nat. Rev. Neurosci. 8, 755-765 (2007).

22. Fraichard, A. et al. In vitro differentiation of embryonic stem cells into glial cells and functional neurons. J. Cell. Sci. 108, 3181-3188 (1995).

23. Pachernik, J. et al. Neural differentiation of pluripotent mouse embryonal carcinoma cells by retinoic acid: inhibitory effect of serum. Physiol. Res. $\mathbf{5 4}$ 115-122 (2005).

24. Bain, G., Ray, W. J., Yao, M. \& Gottlieb, D. I. From embryonal carcinoma cells to neurons: the P19 pathway. BioEssays. 16, 343-348 (1994).

25. Wei, Y., Harris, T. \& Childs, G. Global gene expression patterns during neural differentiation of P19 embryonic carcinoma cells. Differentiation. 70, 204-219 (2002).

26. Endo, M., Antonyak, M. A. \& Cerione, R. A. Cdc42-mTOR signaling pathway controls Hes5 and Pax6 expression in retinoic acid-dependent neural differentiation. J. Biol. Chem. 284, 5107-5118 (2009).

27. Komori, R., Kobayashi, T., Matsuo, H., Kino, K. \& Miyazawa, H. Csn3 gene is regulated by all-trans retinoic acid during neural differentiation in mouse P19 cells. PLoS. One. 8, e61938 (2013).

28. Itoh, F., Nakane, T. \& Chiba, S. Gene expression of MASH-1, MATH-1, neuroD and NSCL-2, basic helix-loop-helix proteins, during neural differentiation in P19 embryonal carcinoma cells. Tohoku. J. Exp. Med. 182, 327-336 (1997).

29. Pratt, M. A., Kralova, J. \& McBurney, M. W. A dominant negative mutation of the alpha retinoic acid receptor gene in a retinoic acid-nonresponsive embryonal carcinoma cell. Mol. Cell. Biol. 10, 6445-6453 (1990).

30. Kruyt, F. A. et al. Transcriptional regulation of retinoic acid receptor beta in retinoic acid-sensitive and -resistant P19 embryocarcinoma cells. Mech. Dev. 33, 171-178 (1991).

31. Zheng, B. et al. Kruppel-like factor 4 inhibits proliferation by platelet-derived growth factor receptor beta-mediated, not by retinoic acid receptor alphamediated, phosphatidylinositol 3-kinase and ERK signaling in vascular smooth muscle cells. J. Biol. Chem. 284, 22773-22785 (2009).

32. Shroot, B. \& Michel, S. Pharmacology and chemistry of adapalene. J. Am. Acad. Dermatol. 36, S96-103 (1997)

33. Harmon, M. A., Boehm, M. F., Heyman, R. A. \& Mangelsdorf, D. J. Activation of mammalian retinoid $\mathrm{X}$ receptors by the insect growth regulator methoprene. Proc. Natl. Acad. Sci. U. S. A. 92, 6157-6160 (1995).

34. Wang, C. et al. Cell aggregation-induced FGF8 elevation is essential for P19 cell neural differentiation. Mol. Biol. Cell. 17, 3075-3084 (2006).

35. Pinheiro, P., Gering, M. \& Patient, R. The basic helix-loop-helix transcription factor, Tal2, marks the lateral floor plate of the spinal cord in zebrafish. Gene. Expr. Patterns. 4, 85-92 (2004).

36. Yang, L., Rastegar, S. \& Strahle, U. Regulatory interactions specifying KolmerAgduhr interneurons. Development. 137, 2713-2722 (2010).

37. Gao, X. et al. A role of $\mathrm{N}$-cadherin in neuronal differentiation of embryonic carcinoma P19 cells. Biochem. Biophys. Res. Commun. 284, 1098-1103 (2001).

38. Niwa, H., Yamamura, K. \& Miyazaki, J. Efficient selection for high-expression transfectants with a novel eukaryotic vector. Gene. 108, 193-199 (1991).

\section{Acknowledgments}

We thank Issei Hamada for technical support. This research was supported by research grant from Tokushima Bunri University, Japan.

\section{Author contributions}

T.K., R.K., K.K. and H.M. conceived the project. T.K., R.K. and H.M. designed the experiments. T.K., R.K. and K.I. performed the experiments. T.K., S.T. and H.M. wrote the manuscript with discussion of all other authors. All authors reviewed the manuscript.

\section{Additional information}

Supplementary information accompanies this paper at http://www.nature.com/ scientificreports

Competing financial interests: The authors declare no competing financial interests

How to cite this article: Kobayashi, T. et al. Tal2 expression is induced by all-trans retinoic acid in P19 cells prior to acquisition of neural fate. Sci. Rep. 4, 4935; DOI:10.1038/srep04935 (2014)

This work is licensed under a Creative Commons Attribution 3.0 Unported License. The images in this article are included in the article's Creative Commons license, unless indicated otherwise in the image credit; if the image is not included under the Creative Commons license, users will need to obtain permission from the license holder in order to reproduce the image. To view a copy of this license, visit http://creativecommons.org/licenses/by/3.0/ 